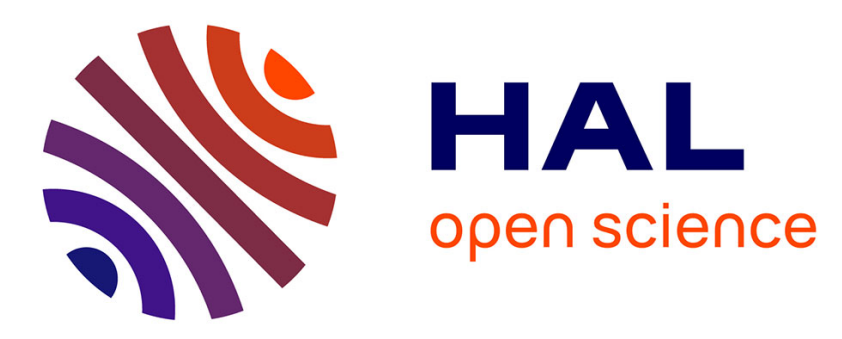

\title{
Study of Viscoelastic Effect on the Frequency Shift of Microcantilever Chemical Sensors
}

Uttara Sampath, Stephen Heinrich, Fabien Josse, Frédéric Lochon, Isabelle Dufour, Dominique Rebiere

\section{- To cite this version:}

Uttara Sampath, Stephen Heinrich, Fabien Josse, Frédéric Lochon, Isabelle Dufour, et al.. Study of Viscoelastic Effect on the Frequency Shift of Microcantilever Chemical Sensors. IEEE Transactions on Ultrasonics, Ferroelectrics and Frequency Control, 2006, 53, pp.2166-2173. hal-00203740

\author{
HAL Id: hal-00203740 \\ https://hal.science/hal-00203740
}

Submitted on 13 Nov 2014

HAL is a multi-disciplinary open access archive for the deposit and dissemination of scientific research documents, whether they are published or not. The documents may come from teaching and research institutions in France or abroad, or from public or private research centers.
L'archive ouverte pluridisciplinaire HAL, est destinée au dépôt et à la diffusion de documents scientifiques de niveau recherche, publiés ou non, émanant des établissements d'enseignement et de recherche français ou étrangers, des laboratoires publics ou privés. 
Preprint - IEEE Transaction on Ultrasonics, Ferroelectrics and Frequency Control, Vol. 53, 2006, pp. $2166-2173$. (doi: 10.1109/TUFFC.2006.156)

\title{
Study of Viscoelastic Effect on the Frequency Shift of Microcantilever Chemical Sensors
}

\author{
Sampath U. ${ }^{1}$, Heinrich S.M. ${ }^{2}$, Josse F. ${ }^{1}$, Lochon F. ${ }^{3}$, Dufour I. ${ }^{3}$, Rebière D. ${ }^{3}$ \\ ${ }^{1}$ Marquette University, \\ Microsensor Research Laboratory and Department of Electrical and Computer Engineering \\ P.O. Box 1881, Milwaukee, \\ WI 53201-1881, USA, \\ fabien.josse@marquette.edu \\ ${ }^{2}$ Marquette University, \\ Department of Civil and Environmental Engineering \\ P.O.Box 1881, Milwaukee, \\ WI 53201-1881, USA, \\ stephen.heinrich@marquette.edu \\ ${ }^{3}$ IXL Laboratory, CNRS UMR5818, \\ ENSEIRB/Université Bordeaux 1, 351 cours de la Libération, \\ 33405 Talence Cedex, France, \\ dufour@ixl.fr
}

\section{Abstract}

Microcantilevers coated with a chemically sensitive layer are increasingly being used in chemical detection systems. The sensitive coating, often a polymer, absorbs specific molecules, which can be detected by monitoring the shift in the mechanical resonant frequency. Usually, the frequency shift resulting from molecular absorption is interpreted as a mass loading effect. However, mass loading is not the only effect that has an impact on the frequency shift; the viscoelastic properties of the sensitive coating are also affected by the sorption process. Sorption-induced modulus changes are typically difficult to characterize. However, it is known that the sorption of analyte molecules in a polymer coating results in the plasticization of the coating. In most cases, the polymer becomes more rubbery with increasing concentration of analyte molecules, i.e., the coating becomes softer with increasing loss modulus while the 
Preprint - IEEE Transaction on Ultrasonics, Ferroelectrics and Frequency Control, Vol. 53, 2006, pp. 2166-2173. (doi: 10.1109/TUFFC.2006.156)

storage modulus decreases. Using a new analytical model developed for the resonant frequency expression of a hybrid microcantilever (elastic base and viscoelastic layer), the effects of the modification of the storage and loss moduli of the sensitive layer on the resonant frequency are examined. The main conclusion of this analytical study is that, even if the sensitive coating moduli are small compared to the base cantilever's Young's modulus, the effect of the change in the viscoelastic coating properties could contribute significantly to the overall frequency shift (8$23 \%$ in the simulations depending on the coating thickness, with even higher contributions for other sets of problem parameters).

\section{INTRODUCTION}

The use of microcantilevers as transducers in chemical sensing systems has increased in recent years. This is due to the projected high sensitivity and low limit of detection (LOD). For chemical detection, the microcantilever is coated with a chemically sensitive layer, which absorbs the molecule of interest. The absorbed molecules can then be detected by monitoring the mechanical resonant frequency. Usually, the frequency shift resulting from molecular absorption is interpreted as a mass loading effect [1-7]. However, in the case of the chemically sensitive polymeric layer, mass loading is not the only effect that has an impact on the frequency shift; the viscoelastic properties of the sensitive coating are also affected by the sorption process. A complete and more accurate model of the frequency shift for a hybrid (elastic/viscoelastic) beam must take into account the contributions of both mass loading and the changes in the viscoelastic properties of the sensitive coating. The aim of this paper is to study the contributions from both the absorbed mass and this "viscoelastic effect" and to compare the relative importance of these effects. 
Preprint - IEEE Transaction on Ultrasonics, Ferroelectrics and Frequency Control, Vol. 53, 2006, pp. 2166-2173. (doi: 10.1109/TUFFC.2006.156)

In section II, a new analytical model for the resonant frequency expression of a hybrid microcantilever (elastic base and viscoelastic layer) is presented. Then in section III the frequency shift due to analyte sorption by the viscoelastic layer is analyzed. It is shown that the frequency shift is composed of three effects: mass loading (added mass) effect, layer elasticity effect, and a neutral-axis shift effect associated with variation in layer properties. Some simulations are presented in section IV and a discussion shows that ignoring the changes in the viscoelastic layer properties may introduce a significant source of error.

\section{RESONANT FREQUENCY OF HYBRID MICROCANTILEVER}

Because of the prevalence of polymer coatings used in resonant microcantilever sensor applications and the viscoelastic properties exhibited by such materials, a natural question arises: what is the impact of the coating's viscoelastic properties on the resonant frequency (and frequency shift) of the microcantilever sensor? In most previous studies, the layer properties have been ignored entirely, an assumption that is usually justified by considering only relatively thin, soft, layers. However, in some applications sensitivity may be increased significantly by using a coating thickness that approaches that of the base layer, in which case a careful consideration of the coating properties is warranted. This is what provides the motivation for the analytical model that is summarized in what follows.

The theoretical model used in the present study is based on the idealized system depicted in Fig. 1. The base layer (often silicon) is assumed to be elastic, while the sensitive coating is taken to be viscoelastic. Using the results of [8], the "hybrid" (elastic/viscoelastic) beam, can be assumed to be replaced by an equivalent homogeneous viscoelastic beam whose complex flexural rigidity, $(E I)^{*}$, is given as 
Preprint - IEEE Transaction on Ultrasonics, Ferroelectrics and Frequency Control, Vol. 53, 2006, pp. 2166-2173. (doi: 10.1109/TUFFC.2006.156)

$$
(E I)^{*}=E_{1} I_{1}+E_{2}^{\prime}(\omega) I_{2}+j E_{2}^{\prime \prime}(\omega) I_{2}
$$

where $E_{1}$ is the Young's modulus of the elastic material and $E_{2}^{\prime}+j E_{2}{ }_{2}$ is the complex Young's modulus of the viscoelastic sensitive layer. $I_{1}$ and $I_{2}$ are the moments of inertia of the elastic and viscoelastic beam layers, respectively:

$$
\begin{gathered}
I_{1}=\frac{b h_{1}^{3}}{12}+b h_{1}\left(h_{N}-h_{2}-\frac{h_{1}}{2}\right)^{2} \\
I_{2}=\frac{b h_{2}^{3}}{12}+b h_{2}\left(h_{N}-\frac{h_{2}}{2}\right)^{2}
\end{gathered}
$$

where $b$ is the microcantilever width, and $h_{1}$ and $h_{2}$ are the microcantilever and sensitive coating thicknesses, respectively. The geometric properties (2) and (3) are with respect to the equivalent fixed neutral axis of the hybrid cross section, whose position relative to the top of the cross section is given by

$$
h_{N}=\frac{h_{2}}{2}+\frac{h_{1} E_{1}}{2} \frac{\left(h_{1}+h_{2}\right)\left(h_{1} E_{1}+h_{2} E_{2}^{\prime}\right)}{\left(h_{1} E_{1}+h_{2} E_{2}^{\prime}\right)^{2}+h_{2}^{2} E_{2}^{\prime 2}}
$$

Note that an exact formulation of the hybrid beam problem would result in a neutral-axis position that varies with time; thus, Eq. (4) may be viewed as a type of "average" position of the neutral axis. This simplification not only results in a tractable problem, but it yields a complex-rigidity formula that is more general than other existing approximations for hybrid cross sections (e.g., the Oberst approximation $[9,10])$, which usually require that the coating is relatively thin and/or soft. (Details may be found in [8].) 
Preprint - IEEE Transaction on Ultrasonics, Ferroelectrics and Frequency Control, Vol. 53, 2006, pp. 2166-2173. (doi: 10.1109/TUFFC.2006.156)

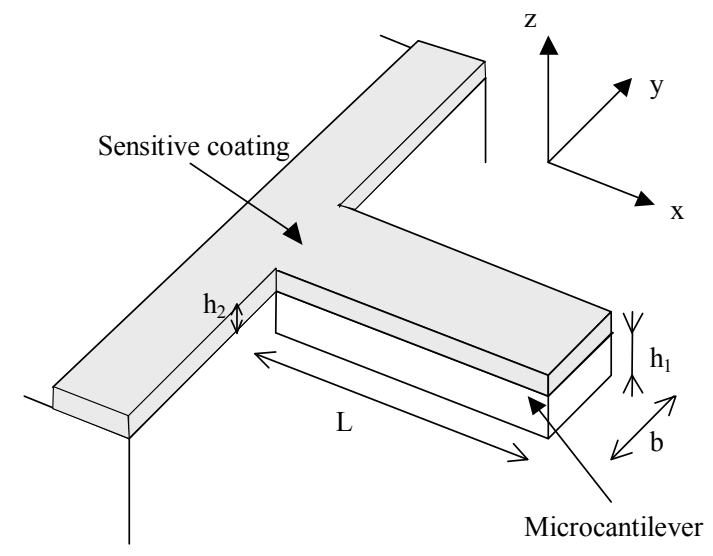

Fig. 1. Geometry of the cantilever and its sensitive coating.

Using the complex flexural rigidity given by (1), the equation of motion of the harmonically excited hybrid beam, considering the only loss mechanism to be associated with the loss modulus of the sensitive layer, takes the well-known form (e.g., [9])

$$
(E I)^{*} \frac{\partial^{4} w(x, t)}{\partial x^{4}}+m_{L} \frac{\partial^{2} w(x, t)}{\partial t^{2}}=F(x) e^{j \varpi t}
$$

where $w(x, t)$ is the transverse displacement, $F(x)$ is the arbitrary distribution of the force amplitude along the length of the beam, $m_{L}$ is the mass per unit length of the beam and $\varpi$ is the forcing frequency. Following standard procedures for solving (5) (e.g., [9]), an expression for the resonant frequency, $f_{\text {res }}$, can be obtained:

$$
f_{\text {res }}=\frac{\lambda_{0}^{2}}{2 \pi L^{2}} \sqrt{\frac{E_{1} I_{1}+E_{2}^{\prime} I_{2}}{m_{L}}}
$$


Preprint - IEEE Transaction on Ultrasonics, Ferroelectrics and Frequency Control, Vol. 53, 2006, pp. 2166-2173. (doi: 10.1109/TUFFC.2006.156)

where $L$ is the microcantilever length and $\lambda_{0}=1.875$ for the first-order mode. Note that in many cases the second term under the radical in (6) is neglected; however, here we wish to examine the importance of this term in detail, specifically how changes in concentration affect the frequency shift. Although the losses due to the environment are neglected in this study, it is expected that, when environmental losses are present, changes in the layer properties will cause a similar relative change in frequency.

In the following section, expression (6) is used as a starting point to study the effect that sorption of analyte has on the frequency shift. In addition, the total frequency shift expression will be partitioned into three contributions, each having a specific physical meaning. The qualitative effect (increase or decrease) that a sorption-induced change in a physical parameter has on frequency shift will also be examined in Section III.

\section{ANALYTE SORPTION INTO THE VisCOELASTIC LAYER}

Analyte sorption into the sensitive coating, which is usually a polymer, results in the modification of its mass but also its complex Young's modulus. Using the partition coefficient of the sensitive layer/analyte pair, it is possible to estimate the mass modification. The sorptioninduced modulus changes are typically more difficult to characterize. However, it is known that the sorption of analyte molecules in the polymer results in the plasticization of the coating [11]. In most cases, the polymer becomes more rubbery with increasing concentration of analyte molecules, i.e. the storage modulus, $E_{2}^{\prime}(\omega)$, decreases and the loss modulus, $E_{2}^{\prime \prime}(\omega)$, becomes larger.

The frequency shift due to analyte sorption, $\Delta f_{\text {res }}$, can be expressed in terms of the analyteinduced variations in the mass, the storage Young's modulus, and the moments of inertia. By 
Preprint - IEEE Transaction on Ultrasonics, Ferroelectrics and Frequency Control, Vol. 53, 2006, pp. 2166-2173. (doi: 10.1109/TUFFC.2006.156)

taking the natural logarithm of both sides of (6), taking the differential of the resulting expression, and assuming that the relevant variations are sufficiently small so that differentials may be replaced by finite variations, the following expression for the relative frequency shift may be derived:

$$
\frac{\Delta f_{\text {res }}}{f_{\text {res }}}=-\frac{\Delta m_{L}}{2 m_{L}}+\frac{I_{2} \Delta E_{2}^{\prime}}{2\left(E_{1} I_{1}+E_{2}^{\prime} I_{2}\right)}+\frac{E_{1} \Delta I_{1}+E_{2}^{\prime} \Delta I_{2}}{2\left(E_{1} I_{1}+E_{2}^{\prime} I_{2}\right)}
$$

The first term on the right-hand side of (7) is the well-known "mass loading effect," while the remaining terms are due to the changes in the viscoelastic layer properties. The second term in (7) represents the "coating elasticity effect," as it is associated only with the change in the coating's elastic storage modulus, $E_{2}^{\prime}$. Note that the second term does not include the effect of $\Delta E_{2}^{\prime}$ on the position of the beam's neutral axis, as evidenced by the fact that $I_{1}$ and $I_{2}$ are held fixed in the second term. The final term corresponds to the "neutral-axis shift effect," because it incorporates the effect of the changing coating properties $\left(E_{2}^{\prime}, E^{\prime \prime}{ }_{2}\right)$ on the neutral axis position. This is reflected in the changes in $I_{1}$ and $I_{2}$ appearing in the third term. (Recall that these are moments of inertia of the individual layer cross sections with respect to the neutral axis.) The influence of these three terms will be analyzed hereafter.

\section{A. Mass Loading Effect}

In (7) the mass loading effect, which historically has been the only effect considered in sensor applications, is given by the term

$$
\left(\frac{\Delta f_{\text {res }}}{f_{\text {res }}}\right)_{m_{L}}=-\frac{\Delta m_{L}}{2 m_{L}}
$$


Preprint - IEEE Transaction on Ultrasonics, Ferroelectrics and Frequency Control, Vol. 53, 2006, pp. 2166-2173. (doi: 10.1109/TUFFC.2006.156)

Due to analyte sorption, the mass of the sensitive coating increases, thereby decreasing the resonant frequency of the microcantilever as indicated in (8). The expression for the mass increase can be estimated if the partition coefficient $K$ of the analyte/coating pair is known:

$$
\Delta m_{L}=K h_{2} b C_{A}
$$

where $C_{A}$ is the analyte concentration in the fluid environment. The partition coefficient $K$ represents the ratio of the analyte concentration in the coating to the analyte concentration in the surrounding fluid.

\section{B. Coating Elasticity Effect}

In (7) the second term represents the effect of the coating's storage modulus (elasticity), assuming that the neutral axis position remains unchanged:

$$
\left(\frac{\Delta f_{\text {res }}}{f_{\text {res }}}\right)_{E_{2}^{\prime}}=\frac{I_{2} \Delta E_{2}^{\prime}}{2\left(E_{1} I_{1}+E_{2}^{\prime} I_{2}\right)}
$$

Due to analyte sorption, the storage modulus of the sensitive layer, $E_{2}^{\prime}$, decreases and consequently expression (10) shows that the resonant frequency decreases, as expected. So this effect is a cumulative effect with the mass loading effect.

For a given polymer and a given analyte concentration in a fluid environment it is very difficult to estimate quantitatively the modification of the Young's modulus of the polymer. However, in the simulations that will be presented in Section IV, a rational means for specifying a reasonable value of $\Delta E^{\prime}{ }_{2}$ will be outlined for a case in which direct experimental data is not available. 
Preprint - IEEE Transaction on Ultrasonics, Ferroelectrics and Frequency Control, Vol. 53, 2006, pp. 2166-2173. (doi: 10.1109/TUFFC.2006.156)

\section{Neutral-Axis Shift Effect}

In (7) the last term is due to the shift in the neutral axis position, and hence the moments of inertia, due to changes in the viscoelastic properties of the coating:

$$
\left(\frac{\Delta f_{\text {res }}}{f_{\text {res }}}\right)_{I}=\frac{E_{1} \Delta I_{1}+E_{2}^{\prime} \Delta I_{2}}{2\left(E_{1} I_{1}+E^{\prime}{ }_{2} I_{2}\right)}
$$

For small variations the changes in the moments of inertia may be written as

$$
\begin{aligned}
& \Delta I_{1}=\frac{\partial I_{1}}{\partial E_{2}^{\prime}} \Delta E_{2}^{\prime}+\frac{\partial I_{1}}{\partial E_{2}} \Delta E_{2}^{\prime \prime} \\
& \Delta I_{2}=\frac{\partial I_{2}}{\partial E_{2}^{\prime}} \Delta E_{2}^{\prime}+\frac{\partial I_{2}}{\partial E_{2}^{\prime \prime}} \Delta E_{2}^{\prime \prime}
\end{aligned}
$$

Using these expressions for $\Delta I_{1}$ and $\Delta I_{2}$ yields

$$
\left(\frac{\Delta f_{\text {res }}}{f_{\text {res }}}\right)_{I}=\frac{E_{1} \frac{\partial I_{1}}{\partial E_{2}^{\prime}}+E_{2}^{\prime}{ }_{2} \frac{\partial I_{2}}{\partial E_{2}^{\prime}}}{2\left(E_{1} I_{1}+E_{2}^{\prime} I_{2}\right)} \Delta E_{2}^{\prime}+\frac{E_{1} \frac{\partial I_{1}}{\partial E_{2}^{\prime \prime}}+E_{2}^{\prime}{ }_{2} \frac{\partial I_{2}}{\partial E_{2}^{\prime \prime}}}{2\left(E_{1} I_{1}+E_{2}^{\prime} I_{2}\right)} \Delta E_{2}^{\prime \prime}
$$

which may be rewritten as

$$
\left(\frac{\Delta f_{\text {res }}}{f_{\text {res }}}\right)_{I}=\alpha \Delta E_{2}^{\prime}+\beta \Delta E^{\prime \prime}{ }_{2}
$$

with 
Preprint - IEEE Transaction on Ultrasonics, Ferroelectrics and Frequency Control, Vol. 53, 2006, pp. 2166-2173. (doi: 10.1109/TUFFC.2006.156)

$$
\begin{gathered}
\alpha \equiv \frac{b h_{1}^{2} h_{2}^{3}\left(h_{1}+h_{2}\right)^{2} E_{1}^{2} E_{2}^{\prime \prime 2}\left(\left(h_{1} E_{1}+h_{2} E_{2}^{\prime}\right)^{2}-h_{2}^{2} E_{2}^{\prime \prime 2}\right)}{4\left(E_{1} I_{1}+E_{2}^{\prime} I_{2}\right)\left(\left(h_{1} E_{1}+h_{2} E_{2}^{\prime}\right)^{2}+h_{2}^{2} E_{2}^{\prime 2}\right)^{3}} \\
\beta \equiv \frac{b h_{1}^{2} h_{2}^{4}\left(h_{1}+h_{2}\right)^{2} E_{1}^{2} E_{2}^{\prime \prime}{ }_{2}\left(h_{1} E_{1}+h_{2} E_{2}^{\prime}\right)}{2\left(E_{1} I_{1}+E_{2}^{\prime} I_{2}\right)\left(\left(h_{1} E_{1}+h_{2} E_{2}^{\prime}\right)^{2}+h_{2}^{2} E_{2}^{\prime 2}\right)^{3}}
\end{gathered}
$$

As mentioned previously, due to analyte sorption the storage Young's modulus of the sensitive layer decreases $\left(\Delta E^{\prime}{ }_{2}<0\right)$. This results in the modification of the neutral-axis contribution to the frequency shift according to $(15,16)$ and implies that $\Delta f_{\text {res }}$ has the same sign as the expression $h_{2}^{2} E_{2}^{\prime \prime 2}-\left(h_{1} E_{1}+h_{2} E_{2}^{\prime}\right)^{2}$. Assuming that the cantilever base material is silicon and the sensitive layer is a polymer, then due to the Young's modulus values of these different materials, the first term of (15) is negative and consequently the decrease in storage modulus causes a decrease in the resonant frequency shift. So this effect (modification of neutral axis position due to the decrease of the storage Young's modulus) is a cumulative effect with the mass loading effect.

Usually, the analyte sorption also modifies the loss modulus of the coating in such a way that $\Delta E{ }_{2}>0$. Using $(15,17)$, it can be seen that the change in this physical parameter causes $\Delta f_{\text {res }}>0$. So, this effect (modification of the neutral axis position due to the increase of the storage Young's modulus) is an opposite effect with the mass loading effect.

\section{Qualitative Effects of Analyte Sorption on Frequency Shift}

Table I summarizes the qualitative effects of analyte sorption on frequency shift that were just discussed. These results are based on (8), (10), and (15), which are predicated upon the assumption of small changes in the relevant quantities. This table clearly shows that, for increasing concentration of analyte (i.e., increasing the mass and loss modulus of the coating, 
Preprint - IEEE Transaction on Ultrasonics, Ferroelectrics and Frequency Control, Vol. 53, 2006, pp. 2166-2173. (doi: 10.1109/TUFFC.2006.156)

decreasing the storage modulus of the coating), the resonant frequency shift due to the mass loading and coating elasticity effects are cumulative. Both result in a decrease in frequency. The neutral-axis shift effect receives a negative contribution from $\Delta E_{2}^{\prime}$ and a positive contribution from $\Delta E_{2}{ }_{2}$, so that a general qualitative conclusion cannot be drawn regarding this effect. However, as will be seen in the next section, the magnitude of the neutral-axis shift effect appears to be extremely small so that it is not expected to be of practical significance.

TABLE I. QUALITATIVE EFFECTS OF ANALYTE SORPTION

\begin{tabular}{|c|c|c|c|c|}
\hline \multirow{2}{*}{ Physical effect } & $\Delta f_{\text {res }}$ due to & $\Delta f_{\text {res }}$ due to & $\Delta f_{\text {res }}$ due to & \\
& mass effect & elastic effect & $\begin{array}{c}\text { neutral axis } \\
\text { effect }\end{array}$ & $\Delta f_{\text {res }}$ total \\
\hline$\Delta m_{L}>0$ & $\left(\Delta f_{\text {res }}\right)_{m_{L}}<0$ & $\left(\Delta f_{\text {res }}\right)_{E_{2}^{\prime}}=0$ & $\left(\Delta f_{\text {res }}\right)_{I}=0$ & $\Delta f_{\text {res }}<0$ \\
\hline$\Delta E^{\prime}{ }_{2}<0$ & $\left(\Delta f_{\text {res }}\right)_{m_{L}}=0$ & $\left(\Delta f_{\text {res }}\right)_{E_{2}^{\prime}}<0$ & $\left(\Delta f_{\text {res }}\right)_{I}<0$ & $\Delta f_{\text {res }}<0$ \\
\hline$\Delta E^{\prime \prime}{ }_{2}>0$ & $\left(\Delta f_{\text {res }}\right)_{m_{L}}=0$ & $\left(\Delta f_{\text {res }}\right)_{E_{2}^{\prime}}=0$ & $\left(\Delta f_{\text {res }}\right)_{I}>0$ & $\Delta f_{\text {res }}>0$ \\
\hline
\end{tabular}

\section{Simulations AND Discussion}

In this section numerical results are presented to quantify the contribution of each effect to the overall frequency shift of a chemical sensor in gas phase, and to determine under what conditions a particular effect may be neglected. Another objective is to investigate the accuracy of the approximate equations (8), (10) and (15). 
Preprint - IEEE Transaction on Ultrasonics, Ferroelectrics and Frequency Control, Vol. 53, 2006, pp. 2166-2173. (doi: 10.1109/TUFFC.2006.156)

\section{A. Simulation Parameters}

For the simulations, the geometry of the microcantilever is given by $L=300 \mu \mathrm{m}, \mathrm{b}=50 \mu \mathrm{m}$ and $h_{1}=4 \mu \mathrm{m}$, giving a nominal resonant frequency of $58 \mathrm{kHz}$. Poly(isobutylene) (PIB) and toluene are assumed here as the coating material and analyte, respectively. The coating thickness is taken as $h_{2}=1 \mu \mathrm{m}$. The partition coefficient for toluene (in gas phase) and PIB, is assumed to be $K=583$ [12-14]. For PIB at the frequency of interest, the storage shear modulus is $G^{\prime}=144 \mathrm{MPa}$ and the loss shear modulus is $G^{\prime \prime}=218 \mathrm{MPa}$ [15]. The Poisson's ratio of PIB is assumed to be $v=0.49$ [16].

The shear moduli are converted to Young's moduli by using the mechanics of materials relationship

$$
G=\frac{E}{2(1+v)}
$$

This expression results in storage and loss Young's moduli for PIB of $E^{\prime}{ }_{2}=429 \mathrm{MPa}$ and $E_{2}=650 \mathrm{MPa}$.

\section{B. Variations in Coating Properties}

The main purpose of this paper is to study the effects of analyte concentration on coating properties and, thus, on frequency response. Hence, it is necessary to specify an appropriate range of coating properties.

As expressed previously, the mass change of the polymer can be estimated using (9) for a given analyte concentration. However, without actual experimental results on the plasticization effect, it is difficult to predict the change in the viscoelastic properties of a polymer due to 
Preprint - IEEE Transaction on Ultrasonics, Ferroelectrics and Frequency Control, Vol. 53, 2006, pp. 2166-2173. (doi: 10.1109/TUFFC.2006.156)

analyte sorption. However, from recent related work using guided SH-SAW sensors [17] changes in the shear modulus of PIB [18] upon exposure to different concentrations of toluene have been experimentally obtained. Due to the approximately linear character of the experimental data, the data may be linearized with respect to concentration, the results of which are summarized here in Table II. This data was obtained for a higher frequency device $(100 \mathrm{MHz})$ and in aqueous solution of the analyte. (For this reason the second column of data was generated using a partition coefficient for toluene/PIB pair in liquid phase of $K=140$ [14].) While such data cannot be utilized directly in the present simulation for a lower-frequency microcantilever device in gas phase, they can serve as a guide for choosing the relative variation in the PIB properties, i.e., the percent change, provided that the variations in property values correspond to the same concentration in the coating.

Note that the experimental data of Table II show that the variations of a polymer's properties due to sorption may be significant, and this may in turn have an important effect on the performance of microcantilever devices. In addition, these changes may be accompanied by swelling effects. Such effects are ignored in the present simulation, but may easily be incorporated by generalizing (11) to account for $\Delta h_{2}$. In what follows, the relative variations of viscoelastic layer properties will be based on an interpolation of the linearized data in Table II. 
Preprint - IEEE Transaction on Ultrasonics, Ferroelectrics and Frequency Control, Vol. 53, 2006, pp. 2166-2173. (doi: 10.1109/TUFFC.2006.156)

table II. Measured Relative Modification of the Young's Moduli of PIB for Toluene SORPTION IN AqueOUS ENVIRONMENT USING GUIDED SH-SAW SENSORS

\begin{tabular}{|c|c|c|c|}
\hline $\begin{array}{c}\text { Toluene } \\
\text { concentration }\end{array}$ & Toluene & & \\
$\mathrm{g} / \mathrm{m}^{3}$ & concentration in & $\%$ Variation in & $\%$ Variation in \\
(ppm aqueous) & $\mathrm{kg} / \mathrm{m}^{3}$ & $E_{2}{ }_{2}(\omega)$ & $E_{2}{ }_{2}(\omega)$ \\
\hline 57.6 & 8.06 & -2.08 & +20.8 \\
$(10)$ & 16.13 & -4.17 & +41.7 \\
\hline 115 & 24.19 & -6.25 & +62.5 \\
\hline$(20)$ & 32.26 & -8.33 & +83.3 \\
\hline 172 & & & +104.1 \\
\hline$(30)$ & 40.32 & -10.42 & \\
\hline 230 & & & \\
\hline$(40)$ & & & \\
\hline 288 & & & \\
\hline
\end{tabular}

\section{Effect of Mass Variation}

Using (8), the relative frequency shift due to a variation in the mass can be calculated. (See Table III). Toluene concentrations of 10,100 , and $1000 \mathrm{mg} / \mathrm{m}^{3}$ in gas have been arbitrarily chosen for studying the effect of adding mass. The corresponding values of toluene concentrations in the coating are calculated directly using the partition coefficient $\mathrm{K}=583$ in (9) and are listed in the first row of the Table III. To facilitate comparison with simulations associated with changing the coating properties, the same values of toluene concentration in the coating will be used in all the numerical results that follow. 
Preprint - IEEE Transaction on Ultrasonics, Ferroelectrics and Frequency Control, Vol. 53, 2006, pp. 2166-2173. (doi: 10.1109/TUFFC.2006.156)

TABLE III. RELATIVE FREQUENCY SHIFT DUE TO MASS VARIATION

\begin{tabular}{|c|c|c|c|}
\hline $\begin{array}{c}\text { Toluene concentration } \\
\text { in coating }\left(\mathrm{g} / \mathrm{m}^{3}\right)\end{array}$ & 5.84 & 58.4 & 584 \\
\hline Toluene concentration & 10 & 100 & 1000 \\
in gas $\left(\mathrm{mg} / \mathrm{m}^{3}\right)$ & $-2.853 \mathrm{e}-$ & $-2.853 \mathrm{e}-$ & $-2.853 \mathrm{e}-$ \\
\hline$\left(\frac{\Delta f_{\text {res }}}{f_{\text {res }}}\right)_{m_{L}}$ & 07 & 06 & 05 \\
\hline
\end{tabular}

Table III shows that the relative frequency shift due to the added mass is linearly related to the concentration. The reason for this is twofold: (a) equation (9) is linear, and (b) a linear approximation (small-variation assumption) was used in deriving (8). The validity of the latter has been confirmed in a separate calculation by showing that the values in Table III are identical to the "exact" theoretical values of frequency shift using (6). While the linearity of the frequency shift due to mass effects is well known in the microcantilever sensors community, Table III is included here because the corresponding results for the other effects (variation in coating elasticity and neutral-axis position) will be presented shortly for comparison purposes.

\section{Effect of Storage Modulus Variation}

The variation of the storage modulus $E_{2}{ }^{\prime}$ has two effects: first, it is responsible for the coating elasticity effect (10); second, it contributes to the neutral-axis shift effect (15). The results of the simulation are shown in Table IV. The first two rows of the table represent the specified concentrations and the corresponding variations in the storage modulus of the coating, while the last two rows show the frequency shifts due to the coating elasticity and neutral-axis shift effects, 
Preprint - IEEE Transaction on Ultrasonics, Ferroelectrics and Frequency Control, Vol. 53, 2006, pp. 2166-2173. (doi: 10.1109/TUFFC.2006.156)

respectively, caused by the change in $E_{2}{ }^{\prime}$. (In (10) and (15), the value of $\Delta E_{2}{ }^{\prime \prime}$ is taken as zero here because we are only considering the effect of $\Delta E_{2}{ }^{\prime}$ in this section.) At the highest concentration $\left(1000 \mathrm{mg} / \mathrm{m}^{3}\right)$, the variation in $E_{2}^{\prime}$ is only $0.15 \%$. However, the table illustrates that these relatively small changes in the storage modulus lead to a frequency shift due to the coating elasticity effect $\left(\frac{\Delta f_{\text {res }}}{f_{\text {res }}}\right)_{E_{2}^{\prime}}$ that is $9 \%$ of that due to the mass loading effect $\left(\frac{\Delta f_{\text {res }}}{f_{\text {res }}}\right)_{m_{L}}$, and that this value $(9 \%)$ is independent of the concentration. Moreover, should the storage modulus of a coating material be more sensitive to analyte concentration than the PIB/toluene combination considered here, the coating elasticity effect could be even more significant relative to the mass effect. In the present case this high sensitivity to such a small change in modulus may seem surprising, until one considers that the corresponding percent change in coating mass is a mere $0.064 \%$. Also illustrated in Table IV is the same linearity of results that was present due to mass variation. The reasons for this are (a) the material property data of Table II is linear with respect to concentration, and (b) a linear approximation was used in deriving (10) and (15). The validity of the second approximation has been confirmed for the values in row 3 of Table IV by performing a separate calculation demonstrating that they are identical to the "exact" theoretical values of frequency shift due to the elasticity effect. However, for the values in the last row of Table IV (neutral-axis shift effect), the same cannot be said. There is approximately $50 \%$ error in the linear estimate at the highest concentration considered. However, because the exact values of the neutral-axis shift effect due to $\Delta E_{2}^{\prime}$ are still 6 to 7 orders of magnitude smaller than the mass and elasticity effects, this issue will not be pursued further. 
Preprint - IEEE Transaction on Ultrasonics, Ferroelectrics and Frequency Control, Vol. 53, 2006, pp. $2166-2173$. (doi: 10.1109/TUFFC.2006.156)

TABlE IV. Relative Frequency Shift due to Storage Young's Modulus Variation

\begin{tabular}{|c|c|c|c|}
\hline $\begin{array}{c}\text { Toluene concentration } \\
\text { in coating }\left(\mathrm{g} / \mathrm{m}^{3}\right)\end{array}$ & 5.84 & 58.4 & 584 \\
\hline$\%$ Variation in $E_{2}^{\prime}(\omega)$ & -0.0015 & -0.015 & -0.15 \\
\hline$\left(\frac{\Delta f_{\text {res }}}{f_{\text {res }}}\right)_{E_{2}^{\prime}}$ & $-2.55 \mathrm{e}-8$ & $-2.55 \mathrm{e}-7$ & $-2.55 \mathrm{e}-6$ \\
\hline$\left(\frac{\Delta f_{\text {res }}}{f_{\text {res }}}\right)_{I}$ & $-5.84 \mathrm{e}-14$ & $-5.84 \mathrm{e}-13$ & $-5.84 \mathrm{e}-12$ \\
\hline
\end{tabular}

\section{E. Effect of Loss Modulus Variation}

The variation of the loss modulus $E_{2}{ }^{\prime \prime}$ modifies the position of the neutral axis and, thus, only the last term of the frequency shift (7), which is isolated in (15). The numerical results are presented in Table V. Analogous to Tables III and IV, this table indicates the percent change in the loss modulus corresponding to the specified concentrations and the corresponding frequency shifts, which in this case are only due to the shifting of the neutral axis. At the highest concentration $\left(1000 \mathrm{mg} / \mathrm{m}^{3}\right)$, the variation in $E_{2}^{\prime \prime}$ is $1.5 \%$, which is 10 times larger than the corresponding variation in storage modulus. However, this change in the loss modulus yields values of the neutral-axis shift effect $\left(\frac{\Delta f_{\text {res }}}{f_{\text {res }}}\right)_{I}$ that are negligible as was the case for changes in the storage modulus. Hence, the total neutral-axis shift effect appears to be negligible in comparison with the mass and coating elasticity effects. Moreover, because changes to the coating loss modulus only affect the neutral-axis shift portion of the frequency change, one may 
Preprint - IEEE Transaction on Ultrasonics, Ferroelectrics and Frequency Control, Vol. 53, 2006, pp. 2166-2173. (doi: 10.1109/TUFFC.2006.156)

conclude that sorption-induced changes to the loss modulus do not appreciably affect the frequency shift of the microcantilever sensor.

TABLE V. Relative FREQUENCY SHIFT DUe to Loss Young's Modulus VARIATION

\begin{tabular}{|c|c|c|c|}
\hline $\begin{array}{c}\text { Toluene concentration } \\
\text { in coating }\left(\mathrm{g} / \mathrm{m}^{3}\right)\end{array}$ & 5.84 & 58.4 & 584 \\
\hline$\%$ Variation in $E_{2}^{\prime \prime}(\omega)$ & 0.015 & 0.15 & 1.5 \\
\hline$\left(\frac{\Delta f_{\text {res }}}{f_{\text {res }}}\right)_{I}$ & $1.94 \mathrm{e}-15$ & $1.94 \mathrm{e}-14$ & $1.94 \mathrm{e}-13$ \\
\hline
\end{tabular}

Also illustrated in Table $\mathrm{V}$ is the same linearity of results that was present due to mass variation and storage modulus variation, and the reasons are identical to those given in the previous section. As in the previous section, the accuracy of the linear approximation (15) that was used to calculate the last row of Table $\mathrm{V}$ is compromised at higher concentrations, but this error will not be considered in more detail because of the negligible magnitude of this contribution to frequency shift.

\section{F. Discussion}

Based on the results generated in the forgoing simulation, a number of potentially important observations may be made.

First of all, the detailed partitioning of the frequency shift into mass, coating elasticity, and neutral-axis shift effects showed that the latter effect is several orders of magnitude smaller than 
Preprint - IEEE Transaction on Ultrasonics, Ferroelectrics and Frequency Control, Vol. 53, 2006, pp. 2166-2173. (doi: 10.1109/TUFFC.2006.156)

the mass loading and coating elasticity effects. Therefore, this effect is expected to be negligible in most, if not all, microcantilever sensor applications.

Of the remaining two effects, the mass loading effect was found to be dominant in the illustrative example, yet the coating elasticity effect also provided a significant contribution to the total frequency shift. In particular, the changes in the coating's storage modulus resulted in a frequency shift that was $9 \%$ of the mass loading effect, which means that the coating elasticity effect represents about $8 \%(=0.09 / 1.09)$ of the total frequency shift. Another simulation performed for a thicker coating $(4 \mu \mathrm{m}$ vs. $1 \mu \mathrm{m})$ resulted in the coating elasticity effect being $30 \%$ of the mass effect, or $23 \%$ of the total frequency shift. Of course, for other values of problem parameters and other analyte/coating pairs, the importance of the change in $E_{2}^{\prime}$ could be even more (or less) important.

At first thought, one might expect that the coating elasticity effect would be less important at smaller concentrations because of the smaller change in coating properties that are expected. However, this analytical study shows that the ratio of the coating elasticity effect to the mass loading effect is independent of concentration. While it is true that coating properties change less at lower concentrations, one must remember that the mass loading is also proportional to the concentration of the sorbed analyte, and this is the reason that the relative importance of the two effects is insensitive to concentration.

The simulations performed herein were based on linearized data relating coating properties to concentration. For the range of concentrations considered, this linearization matches the experimental data quite well. However, the experimental data at higher concentrations shows a nonlinear behavior, i.e., the coating properties begin to change at a higher rate at higher concentrations [17]. This observation means that at higher concentrations (at least for the 
Preprint - IEEE Transaction on Ultrasonics, Ferroelectrics and Frequency Control, Vol. 53, 2006, pp. 2166-2173. (doi: 10.1109/TUFFC.2006.156)

toluene/PIB pair considered) the coating elasticity effect would represent an even more significant contributor to the frequency shift than what was found in this study.

Another implication of this study pertains to the possible experimental determination of partition coefficients of selective coatings using coated microcantilevers. For example, is one were to monitor the frequency shift of a coated cantilever for the purpose of "back-calculating" the partition coefficient of the coating material as was done using surface acoustic wave, an incorrect $K$ value would be obtained if one assumed that the frequency shift were due entirely to the mass loading effect. For example, if in actuality the coating elasticity effect were $30 \%$ of the mass effect, the true $K$ value would be overestimated by $30 \%$. In such a case, the backcalculated $K$ value should be interpreted as an "effective" partition coefficient that lumps the effects of mass loading and coating elasticity (and perhaps other effects, such as swelling) into a single parameter. It would be incorrect to apply the standard mass-based definition of partition coefficient (9) to such a quantity.

A final point of interest is that a very accurate estimate of the reference resonant frequency (prior to analyte exposure) may be made by ignoring the coating entirely. This point is very well known and is often accepted in the calculation of the reference frequency (e.g., [19]). For the problem parameters considered in the simulation of this study, the resonant frequency would be underestimated by only $0.2 \%$ if the coating were ignored in the calculation. However, if the change in the coating elasticity $\left(E_{2}^{\prime}\right)$ were ignored in calculating the frequency shift, the magnitude of the shift would be underestimated by a full $8 \%$, or two orders of magnitude greater than the corresponding error in the reference frequency calculation. For other values of problem parameters, this error could be significantly more. Therefore, it is important to distinguish between (a) the influence that the coating has on the reference frequency (usually negligible) and 
Preprint - IEEE Transaction on Ultrasonics, Ferroelectrics and Frequency Control, Vol. 53, 2006, pp. 2166-2173. (doi: 10.1109/TUFFC.2006.156)

(b) the impact that changes in coating properties may have on the frequency shift (often significant).

\section{SUMMARY AND CONCLUSIONS}

By using a recently developed analytical model for the dynamic behavior of a two-layer hybrid (elastic/viscoelastic) beam, the relationship between a microcantilever sensor's resonant frequency shift caused by analyte sorption and the analyte concentration has been established. Sorption-induced changes in the properties of the selective coating have been included in the model so that their effect can be compared to the mass loading effect that is usually considered. The total frequency shift has been partitioned into three contributions: the mass loading effect, the coating elasticity effect (due to changes in the coating's storage modulus), and the neutral-axis shift effect (caused by changes to the coating's storage and loss moduli).

Based on simulations performed using the model, the following observations are made:

- The neutral-axis shift effect is negligible. This therefore means that any sorption-induced changes in the loss modulus of the coating may be ignored in calculating the frequency shift.

- The coating elasticity effect may be significant. Simulations show that this effect can be up to $30 \%$ of the mass loading effect for a toluene analyte (in gas) and a PIB coating and could possibly be higher for other systems.

- The mass loading and coating elasticity effects are cumulative, i.e., both cause the resonant frequency to decrease.

- For a given sensor system, the ratio of the coating elasticity effect to the mass loading effect is independent of analyte concentration. This result will be valid over a range of 
Preprint - IEEE Transaction on Ultrasonics, Ferroelectrics and Frequency Control, Vol. 53, 2006, pp. 2166-2173. (doi: 10.1109/TUFFC.2006.156)

concentrations in which the storage modulus of the coating varies linearly with concentration. At higher concentrations, nonlinearities in the coating properties may cause the coating elasticity effect to have an even greater influence on the frequency shift.

- Even when detecting a small concentration of analyte which causes very small changes in the coating properties, these changes may still provide a significant contribution to the total frequency shift.

- If a partition coefficient $(K)$ is experimentally determined by monitoring the frequency shift of a coated microcantilever and extracting a $K$ value for the analyte/coating pair, its value may be significantly overestimated if only the mass loading effect is considered. The coating elasticity effect may also contribute significantly to the observed frequency shift, in which case it, too, should be incorporated in the extraction. The analytical model presented in this paper may prove useful for this purpose.

- The common practice of ignoring the coating in calculating the reference frequency of a coated microcantilever is usually justified because the coating is typically much softer than the base material. However, the change in the coating's storage modulus caused by sorption may still have a large impact on the frequency shift, and this effect should therefore be considered.

The main conclusion of this analytical study is that, even if the sensitive coating moduli are small compared to the base cantilever Young's modulus, the effect of the change in the viscoelastic properties (even a very small change) could be a significant increase in the resonant frequency shift beyond that attributed to mass loading only. For chemical sensor applications with detection levels in the pictogram or femtogram range, ignoring this effect could be a significant source of error. 
Preprint - IEEE Transaction on Ultrasonics, Ferroelectrics and Frequency Control, Vol. 53, 2006, pp. $2166-2173$. (doi: 10.1109/TUFFC.2006.156)

\section{ACKNOWLEDGMENTS}

The authors gratefully acknowledge the French Agency for Environment and Energy management (ADEME) and the 'Région Aquitaine' for providing partial sponsorship for this study.

\section{REFERENCES}

[1] P.G. Datskos, T. Thundat, N.V. Lavrik, "Micro and nanocantilever sensors”, Encyclopedia of nanoscience and nanotechnology, Edited by H.S. Nalwa, volume V, pp. 551-560, 2004.

[2] D. Lange, C. Hagleitner, A. Hierlemann , O. Brand and H. Baltes, "Complementary metal oxide semiconductor cantilever arrays on a single chip: mass-sensitive detection of volatile organic compounds”, Analytical Chemistry, vol. 74, pp. 3084-3095, 2002.

[3] A. Gupta, D. Akin and R. Bashir, "Single virus particle mass detection using microresonators with nanoscale thickness”, Applied Physics Letters 84, n¹1, pp 1976-1978, 2004.

[4] Z.J. Davis, G. Abadal, O. Kuhn, O. Hansen, F. Grey and A. Boisen, "Fabrication and characterization of nanoresonating devices for mass detection, Journal of vacuum science and technology", B, 18, n², pp. 612-616, 2000.

[5] T. Ono and M. Esashi, "Mass sensing with resonating ultra-thin silicon beams detected by a double-beam laser Doppler vibrometer", Measurement Science and Technology 15, pp. 1977-1981, 2004.

[6] B.H. Kim, D.P. Kern, S. Raible and U. Weimar, "Fabrication of micromechanical masssensitive resonators with increased mass resolution using SOI substrate", Microelectonic Engineering 61-62, pp. 947-953, 2002. 
Preprint - IEEE Transaction on Ultrasonics, Ferroelectrics and Frequency Control, Vol. 53, 2006, pp. 2166-2173. (doi: 10.1109/TUFFC.2006.156)

[7] E. Forsen, S.G. Nillson, P. Carlberg, G. Abadal, F. Perez-Murano, J. Esteve, J. Montserrat, E. Figueras, F. Campabadal, J. Verd, L. Montelius, N. Barniol and A. Boisen, "Fabrication of cantilever based mass sensors integrated with CMOS using direct write laser lithography on resist”, Nanotechnology 15, pp. S628-S633, 2004.

[8] U. Sampath, "Analytical Modeling of Microcantilever-Based Dynamic Microsensors," M.S. Thesis, Marquette University, Milwaukee, WI, 2005. (available by e-mail request to fabien.josse@marquette.edu)

[9] D.I.G. Jones, “Handbook of Viscoelastic Vibration Damping," John Wiley Publishers, New York, 2001.

[10] D.J. Inman, "Engineering Vibration," Prentice-Hall, Englewood Cliffs, New Jersey, 1996.

[11] J.W. Grate, E.T. Zellers, "The fractional free volume of the sorbed vapor in modeling the viscoelastic contribution to polymer-coated surface acoustic wave vapor sensor responses", Analytical Chemistry, 72, pp. 2861-2868, 2000.

[12] J.W. Grate, "Solubility Interactions and the Design of Chemically Selective Sorbent Coatings for Chemical Sensor Arrays," Sensors and Actuators B, vol 3. pp. 85-111, 1991.

[13] R.A. McGill, "Choosing Polymer Coatings for Gas and Liquid Chemical Microsensors," SPE Society of Plastics Engineers Annual Technical Conference Proc., May 5-10, 1996, pp. 2080-2084, 1996.

[14] Y. Jones, Z. Li, M. M. Johnson, F. Josse, J. Hossenlopp, “ATR-FTIR Spectroscopic Analysis of Sorption of Aqueous Analytes into Polymer Coatings used with Guided SH-SAW Sensors", IEEE Sensors Journal, vol. 5, pp. 1175-1184, 2005.

[15] Ferry, J.D.: Viscoelastic properties of polymers, John Wiley and Sons, 1961. 
Preprint - IEEE Transaction on Ultrasonics, Ferroelectrics and Frequency Control, Vol. 53, 2006, pp. 2166-2173. (doi: 10.1109/TUFFC.2006.156)

[16] Polymer data handbook, Oxford University Press, 1999.

[17] Z. Li, Y. Jones, J. Hossenlopp, R. Cernosek, F. Josse, “Analysis of Liquid-Phase Chemical Detection Using Guided Shear Horizontal-Surface Wave Sensors", Analytical. Chemistry, 77, pp. 4595-4603, 2005.

[18] Martin, S. J.; Frye, G. C.;Senturia, S, D.; "Dynamics and Response of Polymer-Coated Surface Acoustic Wave Devices: Effect of Viscoelastic Properties and Film Resonance", Analytical Chemistry, 66, pp. 2201-2219, 1994.

[19] L. Fadel, C. Zimmermann, I. Dufour, C. Déjous, D. Rebière, J. Pistré, "Coupled determination of gravimetric and elastic effects on two resonant chemical sensors: Love wave and microcantilever platforms", IEEE Transaction on Ultrasonics, Ferroelectrics and Frequency Control, vol. 52, n², pp. 297-303, 2005. 